\section{Questión}

Periodismo / Comunicación ISSN 1669-6581
- Av. $44 \mathrm{~N}^{\circ} 676,1^{\circ}$ piso

CP 1900 - La Plata - Argentina

(i) www.perio.unlp.edu.ar/question

\title{
Aportes para pensar la vulnerabilidad, las políticas y los territorios en tiempos de pandemia.
}

\section{Contributions to thinking about vulnerability, policies and territories in times of pandemic.}

\author{
Vanesa Arrúa \\ Licenciada en Trabajo Social y Magister PLANGESCO (UNLP). \\ Es docente e investigadora en temas de Comunicacion y Politicas Publicas. \\ Coordinó la UEP del Programa Envion en el Ministerio de \\ Desarrollo Social de la Provincia entre 2009 y 2011. \\ Actualmente codirige el CICOPP (FPyCS-UNLP) y \\ trabaja en diferentes proyectos y políticas de juventud. \\ arruavanesa@gmail.com
}

\section{Palabras clave}

Vulnerabilidad - territorio - políticas - políticas sociales

\section{Keywords}

Vulnerability - territory - policies - social policies

\section{Introducción}

Frente a la convocatoria de producir un reflexión escrita acerca del COVID 19, su incidencia planetaria y el modo en que desde estas tierras transitamos este presente, lo primero que me surge es una intuición, la idea de que este momento es muy difícil de ser comprendido desde la inmediatez; es la sensación de no poder trascender la coyuntura de un presente permanente que marca un cambio radical en los modos de organización de las relaciones sociales, y que su verdadero alcance sólo podrá ser sintetizado con el correr del tiempo... es decir, tengo la sensación de que esto recién empieza. 
En este marco me propongo compartir una reflexión sobre un aspecto parcial de toda esta realidad, pero una parcialidad situada. Digo un interés parcial porque me importa una lectura sobre el concepto de vulnerabilidad, noción que algunos sectores de la sociedad a través de los medios de comunicación, utilizan como argumento para legitimar los modos en que el Estado interviene en las comunidades desde diferentes Políticas Públicas y en particular desde las Políticas Sociales.

Esto me acerca al objetivo del artículo, que es situar la reflexión sobre los modos en que la vulnerabilidad es concebida en la gestión de políticas sociales, pensadas como un modo de intervención del Estado, en contextos de COVID 19.

En este marco dos cuestiones aparecen como necesarias, una cuestión ya dicha, vinculada a lo conceptual, y una segunda cuestión que nos permita situarnos en el contexto del devenir de las Políticas Públicas antes de la irrupción de la pandemia como factor de reconfiguración de los modos de relación en la vida cotidiana de las personas.

En relación a la noción de vulnerabilidad, no sólo se trata de un concepto asociado a procesos sociales, también veremos que se ha reflexionado desde los procesos de producción de subjetividad. Por último, me interesa en particular pensar el concepto asociado a un campo de reflexión y producción de Políticas Públicas: la gestión de riesgo.

En este contexto particular entiendo que la gestión de riesgo, como propuesta que permite anticiparse a la atención en contextos de crisis o catástrofes, nos brinda algunos elementos para comprender y dar complejidad a la noción de vulnerabilidad.

\section{Algunas cuestiones sobre el contexto, en el que hablamos de vulnerabilidad}

Creo necesario para situar la reflexión conceptual, recuperar una caracterización general del contexto de gestión de las Políticas Social y las Políticas Públicas en nuestro país en el momento en que, la pandemia producida por el COVID 19, genera la toma de decisiones del Gobierno Nacional, que re organizan la vida cotidiana de toda la población.

Quiero enunciar sólo algunos puntos más relevantes:

- Los antecedentes de precarización del Estado, las Políticas Sociales e general y las de Salud en particular. Hemos atravesado en los últimos cuatro años de gestión del Gobierno de Cambiemos a nivel nacional y provincial, un plan muy efectivo de reducción del Estado en su rol de garante de Derechos Sociales. 
- Como consecuencia de este plan sistemático el Estado Nacional y el Estado Provincial se han empobrecido, desfinanciando las políticas sociales, educativas y de salud y generando además una situación de endeudamiento crítico.

- En términos sociales, se dieron procesos abruptos de empobrecimiento de la población, y precarización del trabajo, con indicadores claros de crecimiento de la desocupación junto al inicio de la recesión económica. Según datos del INDEC, para el cuarto trimestre de 2019 la tasa de desocupación alcanzó el 8,9\%i. Además, el CIPPEC sostiene que casi 2 de cada 10 jóvenes están desempleados (19,3\%)ii.

- El empobrecimiento general, ha afectado particularmente a niños y jóvenes. Ya en 2018, el Observatorio de la Deuda Social de la UCA iii, indicaba que el $51,7 \%$ de la población infantil Argentina vivía bajo la línea de pobreza.

- Un contexto de crecimiento de las violencias de género, con 327 femicidios durante el 2019iv y un promedio de una muerte por día durante los primeros meses del 2020.

- En este contexto asume un gobierno de corte popular cuyas principales prioridades fueron establecidas estratégicamente en tres grandes líneas de acción: renegociación de la deuda externa; lucha contra el hambre y protección de las personas adultas mayores.

Endeudamiento público, precarización del trabajo, crecimiento de la pobreza, hambre, violencias de género, son los principales problemas sociales, de la realidad social y política en la que el COVID hace su aparición en la realidad Argentinav.

Frente a esta situación, y sobre todo, en las últimas semanas, en que la incidencia de la Pandemia toma rasgos clasistas y comienza a expandirse territorialmente en las zonas urbanas de mayor precariedad, es que la noción de vulnerabilidad comienza a tomar relevancia.

En este contexto, es necesaria la reflexión que permita ver que tan profundo es el proceso de la vulnerabilidad social y por qué es necesario trascender lo sanitario para superar los desafíos que el covid 19 impone.

\section{Vulnerabilidad Social}

A fines de los años 90, el sociólogo francés Robert Castel publica "La metamorfosis de la Cuestión Social", libro en el que trabaja desde un enfoque particular, el modo en que el trabajo 
en las sociedades modernas ha funcionado como un organizador, no sólo de las relaciones de producción, sino más bien, de los modos en que las personas lograban un determinado rol, es decir un modo de inscripción en el mundo social.

Este libro es escrito en Francia, en un contexto en el que los asalariados ven cada vez más precarizadas sus condiciones laborales, y grandes mayorías de trabajadores engrosan los índices de desocupación. En palabras del autor la situación era descripta con rasgos que aún tienen vigencia "...marcada por una conmoción que recientemente ha afectado a la condición salarial: el desempleo masivo y la precarización de las situaciones de trabajo, la inadecuación de los sistemas clásicos de protección para cubrir estos estados, la multiplicación de los individuos que ocupan en la sociedad una posición de supernumerarios, "inempleables", desempleados o empleados de manera precaria, intermitente. Para muchos el futuro tiene el sello de lo aleatorio...."vi

Uno de los principales aportes que realizó el autor a la reflexión de los procesos sociales y a las transformaciones en el mundo del trabajo, y de la cuestión social, fue una serie de conceptos que permitieron en aquel momento enunciar la caída de grandes sectores de la población hacia una nueva situación de empobrecimiento y precarización de sus modos de vida.

Los conceptos de filiación social o enrolamiento social, desafiliación, y vulnerabilidad pensados en relación, permiten explicar el tránsito de grandes mayorías desde una zona de integración social plena, dada por un rol social que brinda el trabajo, como espacio de adscripción al mundo social, y un conjunto de lazos y vínculos que esta relación genera, por ejemplo el acceso a la seguridad social, los beneficios de la sindicalización y las oportunidades de desplegar en la vida familiar un potencial de realización de sus integrantes.

Desde esta posición de inclusión plena, la pérdida del trabajo, no sólo significa una pérdida en relación a las posibilidades económicas y de consumo, sino más bien, el deterioro de un conjunto de relaciones de pertenencia y oportunidades en términos de proyecto de vida; que como ya se dijo, trae a "lo social" la inscripción en el mundo del trabajo.

“...Al principio, teníamos (y seguimos teniendo) la intención de explicar la incertidumbre de los estatutos, de la fragilidad del vinculo social, de los itinerarios cuyas trayectoria se ve estremecida...se trata de una reflexión sobre condiciones de la cohesión social a partir del análisis de situaciones de disociación. El objetivo era (y sigue siendo) calibrar este nuevo dato 
contemporáneo: la presencia, se diría que cada vez más insistente, de individuos ubicados como situación de flotación en la estructura social, que pueblan sus intersticios sin encontrar allí un lugar asignado..."vii

En este sentido, para Castel la vulnerabilidad es una situación en la que los sujetos se encuentran, situación caracterizada por la precarización de los vínculos de pertenencia e inscripción en lo social, desde un rol que también es económico y productivo; pero no sólo eso, sino que además se precariza toda la trama de vínculos y relaciones que le ofrece al sujeto trabajador su vínculo con el mundo del trabajo: el acceso a servicios de salud, el ocio en el club o la quinta sindical, la actividad deportiva con compañeros, las vacaciones pagas en hotelería gremial, el acceso a la vivienda, es decir, todo lo que ofrece la condición de trabajador estable para el desarrollo y organización de la vida cotidiana.

“... No obstante en el intento de superar la mera descripción empírica de estas situaciones, he encontrado que el análisis de una relación con el trabajo (o con ausencia del trabajo, o con el atrabajo aleatorio) representaba un factor determinante para reubicarlas en la dinámica social que las constituye. No encaro aquí el trabajo en tanto que relación técnica de producción, sino como un soporte privilegiado de inscripción de la estructura social. Existe, en efecto, -se lo verificará en el largo término- una fuerte correlación entre el lugar que ocupa en la división social del trabajo y la participación en las redes de sociabilidad y en los sistemas de protección que "cubren" a un individuo ante los riesgos de la existencia. De allí la posibilidad de construir lo que yo llamaría metafóricamente "zonas" de cohesión social. Entonces, la asociación "trabajo estable/inserción relacional sólida" caracteriza una zona de integración. A la inversa, la ausencia de participación en alguna actividad productiva y el aislamiento relacional conjugan sus efectos negativos para producir la exclusión, o más bien, cómo trataré de demostrarlo, la desafiliación. La vulnerabilidad social es una zona intermedia, inestable, que conjuga la precariedad del trabajo y la fragilidad de los soportes de proximidad..."viii

Esta descripción, de lo que el autor denomina espacio de integración social sólida, permite reconocer lo que se deteriora, y la descripción de la nueva situación a la que se llega a partir de la pérdida de los lazos de filiación social (no sólo vinculados al trabajo, sino a todo el entorno de relaciones que la filiación habilita).

Para el autor, la vulnerabilidad es la situación en la que las condiciones de vida se precarizan. La incertidumbre ligada a la situación laboral es la puerta de entrada a un proceso de deterioro 
de condiciones de vida, que sin oportunidades de anclar en lo social, desencadena en la ruptura de vínculos con una trama mayor de pertenencia. -

Para el autor es clave detenerse en las relaciones que existen ente vulnerabilidad social y precariedad económica. Entre precariedad económica y la inestabilidad social.

"El modelo propuesto no es estático. Se trata menos de ubicar individuos en estas zonas que de aclarar los procesos que los llevan de una zona a la otra, por ejemplo a parar de la integración, a la vulnerabilidad, o a caer desde la vulnerabilidad a la inexistencia social o desafiliación"ix.

Este marco de reflexión pone de relieve en la escena social los espacios de articulación que hacen que la trama sea más densa, más integrada.

Cuando se habla de población en situación de vulnerabilidad, en contextos de pandemia, las organizaciones sociales de base, la comunidad organizada, cobra relevancia y protagonismo. Es el espacio clave para fortalecer y consolidar tramas de relaciones que eviten el tránsito hacia la desafiliación social.

Las intervenciones ya no pueden ser pensadas en forma de asistencia directa solamente. Los operativos de salud, de controles tendientes a detener la expansión del virus en territorios densamente poblados y con claro déficit habitacional deben ser complementados con estrategias que den protagonismo a las redes políticas de organización social, que históricamente se ocupan en estrategias de "cuidado" de los vecinos.

Es imprescindible que las estrategias barriales que despliega el Estado y las Políticas Públicas se articulen con las organizaciones sociales, porque de lo contrario, la operatoria no hace más que reforzar la situación de precarización del vínculo social, es decir, refuerza la situación de vulnerabilidad.

Se trata de evitar los procesos de desafiliación que conducen a las personas a situaciones de desconexión con los sistemas de protección y cuidado que la comunidad crea para sí misma. Y se trata también de promover los procesos que habilite a los actores de la comunidad a un ejercicio del poder de organización, del cuidado y del trabajo con los otros.

\section{Vulnerabilidad y Subjetividad}

Cómo un modo de complejizar la reflexión sobre el concepto de vulnerabilidad, y en contexto en que problematizar lo social nos conduce al ámbito de lo cotidiano, de la proximidad, dónde 
las relaciones se vuelven sensibles y el contexto de encierro desafía las "resistencias" emocionales, vinculares, subjetivas, cobra relevancia pensar la relación entre vulnerabilidad social y subjetividad.

En este punto surgen varios interrogantes, ¿qué pasa con los sujetos en situación de vulnerabilidad? ¿Hasta dónde la situación de precariedad, que en principio es externa al sujeto se vuelve proceso interno? ¿Se precariza la subjetividad producto de una situación social de vulnerabilidad? ¿Puede una subjetividad vulnerada cuidar a los otros y cuidar de sí misma?

Esta dimensión nos conecta con aspectos de la complejidad social en el ámbito de lo doméstico, en los hogares. La relación entre subjetividad vulnerada y violencia es un aspecto central para comprender aspectos subjetivos de la situación de vulnerabilidad. En líneas generales, al hablar de violencias hago referencia a diferentes tipos de violencias y abusos contra la niñez y la condición de género de las personas.

Retomando el concepto de desafiliación de Castel, es importante no asumirlo cómo algo dado, un hecho que se confirma como ruptura. En este sentido es fundamental trazar un recorrido. El concepto pertenece al mismo grupo de ideas que refieren a la disociación, la descalificación o la invalidación social. Esto significa que para llegar a la desafiliación, el sujeto ha recorrido procesos de disociación, invalidación y descalificación. Estos procesos generan un gran deterioro subjetivo que en muchos casos se expresan y se entrelazan en situaciones de violencias que son vividas en diferentes ámbitos.

Los procesos de subjetivación en contextos de vulnerabilidad social impiden la identificación de algunas cuestiones claves de la vida en sociedad que hacen a un desarrollo subjetivo plenox. El goce de derechos es un factor esencial en la producción de subjetividades de poder. $O$ al menos, saberse y sentirse digno de un derecho que se le es negado, genera procesos de movilización, reclamo y lucha social que dialogan a nivel subjetivo con la relación erótica o thanática que el sujeto tiene con sí mismo.

Esta dimensión política del desarrollo de la subjetividad es clave para comprender procesos de subjetivación y empoderamiento que le permiten a los sujetos la transformación de sus condiciones de vida.

Efron nos acerca en una reflexión sobre la subjetividad adolescente, la descripción sobre el despliegue relacional que la configura como proceso y como trayectoria de vida. El autor considera a la subjetividad como la "forma de existencia de los sujetos"xi. 
La subjetividad se configura en la trama vincular y adquiere relieve porque emerge como singularidad. Las miradas disciplinares encuentran oportunidad de dialogar en la práctica. Las instituciones, y los territorios están además organizados en muchos casos, desde las Políticas Públicas y por miradas disciplinares que dialogan saberes para definir estrategias y acciones. Estos espacios son claves para comprender y abordar las tramas complejas y dinámicas de la subjetividad.

Siguiendo la reflexión de Efron, "Los saberes, las disciplinas que se refieren a ella (la subjetividad) deben reconocer que lo hacen desde un cierto recorte, desde una cierta parcialidad. Es en el entrecruzamiento de los saberes, las disciplinas, que se va armando una concepción dinámica de la subjetividad, pensada como lo no dado, como lo no estático, como en proceso de estructuración y de construcción. De ahí la idea de subjetivación, de una subjetividad que se hace".xii

Dos ideas interesantes de este párrafo, la mirada compleja sobre el concepto de subjetividad, la claridad para enunciar que los saberes disciplinares son provisionales, limitados, incompletos, justifica la necesidad de pensar en diálogos de saberes entre las diferencias disciplinares, planteando nuevos procesos ontológicos acerca de la subjetividad en los procesos de intervención.

En segundo lugar la subjetividad enunciada como proceso que va configurando un modo de relación del sujeto con el mundo, relación que a la vez lo condiciona, lo interpela y modifica. Relación dialógica que va dando forma y estructura, a la vez que permite nuevas formas y re estructura. Sedimentos de relaciones que van cimentando experiencias de poder o no poder. Subjetivación es subjetividad en movimiento a partir de la relación con "los otros".

Esos otros que traducen un mundo al que se está habilitado para entrar y ser parte, o no. Y a la vez, siempre hay un mundo de pertenencia. La presencia del "otro" es inevitable en la configuración subjetiva, porque de esta depende.

El otro, representado en un comienzo por los padres, pero que con el correr del tiempo se va diversificando y encarnando en otras figuras, otras personas, otras instituciones. En síntesis: la subjetivación tiene dos articuladores esenciales: la idea de "en construcción" y la presencia del "otro".xiii

Si el proceso de subjetivación desconoce el goce de Derechos, la subjetividad se vulnera. Es aquí donde el Estado y las Políticas Sociales cumplen un rol clave. Son los actores de la 
Política quienes, frente a contextos de vulnerabilidad social despliegan estrategias para el acceso a derechos. De este modo, el Estado, y los actores que lo representan, se convierten en el "otro" que favorece el despliegue de recursos de subjetividad que permite transitar procesos de empoderamiento y transformación. Condición necesaria e imprescindible para salir de contextos y relaciones de violencia.

La subjetivación es descripta como movimiento en la trama subjetiva. Dinámica de relación que a la vez que transforma la subjetividad, transforma las relaciones. Movimiento y trama como características que hablan de lo múltiple y singular, lo provisorio y permanente, de lo sostenido y lo que sostiene, de la organización y lo organizado. Este aspecto del abordaje desde la subjetividad no puede quedar fuera de estrategias de contención y atención en contextos de pandemia.

\section{Vulnerabilidad y Riesgo}

El concepto de vulnerabilidad es trabajado también desde la mirada que piensa los procesos de Gestión del Riesgo y la atención de los desastres. Este pensamiento tiene clara incidencia en la definición de políticas tendientes a contener y mitigar los efectos de crisis desatadas por eventos naturales o por acciones negligentes de los hombres.

Cardona define la Gestión del Riesgo como "un complejo proceso social cuyo fin último es la reducción de los riesgos y la disminución del impacto de los desastres, partiendo del supuesto de que el riesgo, como proceso social, es dinámico y susceptible a control."xiv

La mirada desde la Gestión de Riesgo permitió complejizar la noción de desastre al considerar las posibilidades de acción que existen para evitar estas situaciones. Desde este enfoque se considera el desastre como la materialización del riesgo, por lo tanto es posible gestionar el riesgo para evitar el desastre, mediante la prevención y la mitigación. De este modo, aún cuando no haya ocurrido un desastre, el riesgo, y sus factores causantes, están presentes y pueden ser modificados $x \mathrm{v}$.

En este contexto la noción de vulnerabilidad es pensada frente a una situación de amenaza, y la relación entre ambos conceptos da por resultado la situación de riesgo en que encuentra una determinada población.

Es decir que la definición de vulnerabilidad está asociada a las condiciones y recursos con los que cuenta una población para enfrentar una amenaza. Podríamos decir entonces que cuantos 
más recursos disponibles en una sociedad para la atención de los afectados por el virus, menor vulnerabilidad de la población frente al riesgo de muerte que provoca el COVID 19.

Otro modo de pensarlo sería que frente a la amenaza de contagio, mayores condiciones para el aislamiento social provocan una disminución del riesgo de contraer la enfermedad. Es en este último sentido que el Gobierno Nacional ha orientado la definición de las políticas que complementan el decreto de aislamiento social preventivo y obligatorio.

La gestión del riesgo, abordada desde su dimensión territorial comprende una complejidad de procesos, dando relevancia a diferentes dimensiones como la social, la histórica, la política, la económica, etc. y detiene la atención en los procesos socioculturales que intensifican o reducen la incidencia del riesgo. En este marco, conceptos como amenaza, vulnerabilidad y riesgo, cobran nuevo sentido a la luz de los enfoques de complejidad, y redefinen los modos de abordaje.

Desde este punto de vista, la situación de vulnerabilidad en contextos de COVID 19, está asociada directamente con las condiciones para garantizar el asilamiento, esto es tener la posibilidad de contar con una vivienda que contenga un hogar seguro (libre de violencias), acceso al alimento, a los servicios en general y a las redes de internet en particular.

Siguiendo a Cardona, el autor advierte que desde los diferentes paradigmas que han trabajado la noción de riesgo, la mirada estructuralista tiene una tendencia a confundir como sinónimo, pobreza con vulnerabilidad, y no a aquella como un factor de ésta. Por otro lado, el enfoque holístico o sistémico, propone conocer y analizar el riesgo, como resultado de una lectura compleja de los modos de organización de los elementos que configuran la realidad a conocer y la dinámica de sus relaciones. Es decir, los modos en que los sistemas de relación organizan la realidad. Para Cardona, la concepción de la realidad en tanto sistema complejo y el conocimiento de los modos en que ese sistema se organiza es clave para la clave en la gestión del riesgo. De este modo, el análisis de la situación de vulnerabilidad en la que se encuentra una determinada población, no es generalizable, sino más bien situada en contextos, procesos y relaciones particulares.

En la gestión territorial, conocer la amenaza es tan importante como conocer la situación de vulnerabilidad en la que se encuentra la población frente a la amenaza, y en este sentido, la comunidad es clave como fuente de información que permite identificar las situaciones particulares que dan viabilidad a las estrategias de reducción de riesgo. 
La gestión del riesgo puede entenderse como un conjunto de acciones dirigidas a la reducción del riesgo (prevención y mitigación), así como la atención del desastre (respuesta, rehabilitación y reconstrucción), en el que se le da un papel predominante al primer aspecto. La gestión territorial del riesgo implica las siguientes tareas:

Estimación del riesgo: En esta tarea se debe trabajar de forma interdisciplinaria para alcanzar no sólo el conocimiento de la amenaza, sino su graduación y diferencias, según los distintos territorios, para realizar estimaciones del nivel de vulnerabilidad y las valoraciones espaciales del riesgo. En estas instancias, las poblaciones pueden jugar un papel fundamental al sumarse a los investigadores a través de procesos de participación ciudadana, lo que redundará en una más apropiada percepción del riesgo.

Disminución del riesgo: En esta tarea se enmarcan las actividades relacionadas con la propuesta y ejecución de medidas de prevención y de mitigación, a partir de los resultados obtenidos en la fase anterior.

Manejo del desastre: En este caso, la labor se divide en dos momentos: ex ante (acciones dirigidas a crear una cultura de la prevención, trabajando para aumentar la resiliencia de las poblaciones en riesgo; y otro, relacionado a todas las acciones propias de la atención de la emergencia, realizadas con el propósito de minimizar las pérdidas.

Estas diferentes instancias del proceso de gestión del riesgo en los territorios, nos dan una visión sobre el modo en que se han orientado las políticas públicas para enfrentar la pandemia en el territorio Nacional. En este contexto el desafío es gestionar en condiciones de distanciamiento, y construir lazos a partir de mediaciones tecnológicas que preserven el aislamiento.

\section{El trabajo desde las políticas y los territorios en contextos de aislamiento}

El gran desafío en la construcción territorial de las políticas es superar las condiciones de aislamiento y gestionar con distanciamiento social, maximizando los cuidados mutuos.

Por otro lado, pensar en el desarrollo de políticas sociales que tiendan a disminuir la situación de vulnerabilidad de la población, requiere identificar una gran diversidad de territorios y situaciones en hogares y organizaciones. En este sentido, las políticas sociales surgidas desde los gobiernos locales son estratégicas para pensar modalidades de intervención. 
Si bien el ámbito local es el indicado para la identificación de las prioridades y el diseño e implementación de las estrategias, estos procesos no pueden pensarse de forma aislada de las políticas definidas en niveles más amplios (provinciales y nacionales) que orientan, contienen, norman y habilitan recursos. A la vez, los diálogos entre los espacios locales, provinciales y nacionales son claves para retroalimentar el estado de situación general, que permite pensarnos en contexto.

La situación de riesgo de contagio frente al COVID 19 y el marco general de aislamiento social, preventivo y obligatorio genera grandes desafíos para atender condiciones de vulnerabilidad signadas por la ruptura de lazos sociales y las condiciones de deterioro subjetivo que supone una situación de aislamiento en contextos de violencia.

En este marco, es indispensable definir y regla mecanismos que permitan distinguir núcleos problemáticos según tipos de vulnerabilidad y garantizar el acceso a bienes y recursos que fortalezcan condiciones de vida, en términos de despliegue de potenciales e intereses, así como contribuir a consolidar la trama vincular de proximidad familiar y aquellas que requieran apoyatura tecnológica como mediación.

En el mismo orden, es muy importante pensar el rol de los medios de comunicación como actores claves en la construcción de condiciones de vida que disminuyan la vulnerabilidad frente a la situación de aislamiento.

En la relación Medios de Comunicación / Políticas Sociales, el acceso a las tecnologías se convierte en un recurso de protección tan necesario como la alimentación. Pensar el acceso a las tecnologías además como medicaciones de acompañamiento, protección y socorro; o cómo factores de consolidación de vínculos socio-afectivos; cómo acceso a consumos culturales, la educación y al conocimiento, es identificarlos con el acceso a Derechos Sociales.

Por otro lado, se trata de horizontalizar las relaciones y la conexión entre efectores del Estado y los destinatarios de las políticas, a través de dispositivos que preservando la distancia social permitan sostener diálogos para un mayor acompañamiento de las situaciones de vulnerabilidad subjetiva requieran.

La articulación con las organizaciones sociales es clave para la agilizar la circulación del conocimiento disponible sobre el territorio, identificando prioridades de acción y construyendo criterios de intervención que permitan una articulación real con la comunidad y viabilicen no 
sólo el acceso a los recursos sino los procesos de transformación que favorezcan un tránsito armónico por el proceso de aislamiento social.

En este contexto las Políticas Sociales deben articular a los procesos políticos una dimensión pedagógica, que permita actualizar permanentemente los sentidos de las decisiones que se toman en contextos de transformación extremadamente dinámicos. Es indispensable la articulación de los procesos de organización política con las comunidades porque desde la dimensión política se discute la toma de decisiones en torno a los objetivos y los recursos; mientras que la dimensión socioeducativa, permite construir consensos respecto de los roles y las modalidades de acción, multiplicando los sentidos que las fundamentan.

Los procesos de gestión integral del riesgo, requieren involucran a los medios de comunicación desde la dimensión política para que se asocien a los procesos socioeducativos. En este sentido, las Políticas de Comunicación Social adquieren un rol estratégico, no sólo en las mediaciones pensadas en términos sociales y comunitarios, sino en la reflexión acerca del rol de los Medios de Comunicación.

Como actores sociales privilegiados con acceso a la mayoría de los hogares, las Políticas Sociales deben articular también desde los medios de comunicación los criterios de intervención que dan sentido a las acciones que realizan, y fundamentalmente trabajar para que desde estos espacios no se contribuya a profundizar las situaciones de vulnerabilidad social o subjetiva que padecen sus destinatarios.

\section{Referencias bibliográficas}

Robert Castel. "La metamorfosis de la cuestión social. Una crónica del asalariado."Editorial PAIDOS, Buenos Aires, 1997) P. 13

Gabriel Kessler. Adolescencia, Pobreza, Ciudadanía y Exclusión. En Adolescencia, pobreza, educación y trabajo. Irene Konterllnik y Claudia Jacinto. (Compiladoras) Editorial Losada y UNICEFF. Buenos Aires. 1997

Rubén Efron, Subjetividad y Adolescencia. En Adolescencia, pobreza, educación y trabajo. Irene Konterllnik y Claudia Jacinto. (Compiladoras) Editorial Losada y UNICEFF. Buenos Aires. 1997 
Omar Darío Cardona. "La necesidad de pensar de manera holística los conceptos de Vulnerabilidad y Riesgo. Una crítica y una revisión necesaria para la gestión. Centro de Estudios sobre Desastre y Riesgo (CEDERI). Universidad Andina. Bogotá, 2001

\section{Referencias en páginas y periódicos virtuales}

Informe del INDEC - 26/03/20 - https://www.indec.gob.ar/indec/web/Nivel4-Tema-4-31-58

Centro de Implementación de Políticas Públicas para la equidad y el crecimiento -CIPPEChttps://www.cippec.org/textual/la-argentina-es-el-pais-con-mayor-desempleo-juvenil-de-laregion/

Infobae Económico. 7 de Junio de 2019. https://www.infobae.com/economia/2019/06/07/masde-la-mitad-de-los-ninos-de-argentina-son-pobres-segun-un-estudio-de-la-uca/

Datos del Observatorio "Ahora si que nos ven", tomados por Perfil en: https://www.perfil.com/noticias/actualidad/femicidios-2019-observatorio-ahora-que-si-nos-venpublico-hubo-1-cada-24-horas.phtml

\section{Notas}

i Informe del INDEC - 26/03/20 - https://www.indec.gob.ar/indec/web/Nivel4-Tema-4-31-58

ii Centro de Implementación de Políticas Públicas para la equidad y el crecimiento -CIPPEChttps://www.cippec.org/textual/la-argentina-es-el-pais-con-mayor-desempleo-juvenil-de-la-region/

iii Infobae Económico. 7 de Junio de 2019. https://www.infobae.com/economia/2019/06/07/mas-de-la-mitad-de-losninos-de-argentina-son-pobres-segun-un-estudio-de-la-ucal

iv Según datos del Observatorio "Ahora si que nos ven", tomados por Perfil en: https://www.perfil.com/noticias/actualidad/femicidios-2019-observatorio-ahora-que-si-nos-ven-publico-hubo-1-cada-24horas.phtml

$\checkmark$ Durante el mes de marzo, frente a la decisión del Gobierno Nacional de pagar un subsidio a familias que vieran afectados sus ingresos debido a las medidas de aislamiento social preventivo y obligatorio, 11 millones de personas se inscribieron para cobrar el Ingreso Familiar de Emergencia.

vi Robert Castel. "La metamorfosis de la cuestión social. Una crónica del asalariado."Editorial PAIDOS, Buenos Aires, 1997) P. 13

viiop. Cit. P.15

viii Op. Cit. P.15

ix Op. Cit. P16

× Gabriel Kessler. Adolescencia, Pobreza, Ciudadanía y Exclusión. En Adolescencia, pobreza, educación y trabajo. Irene Konterllnik y Claudia Jacinto. (Compiladoras) Editorial Losada y UNICEFF. Buenos Aires. 1997

xi Rubén Efron, Subjetividad y Adolescencia. En Adolescencia, pobreza, educación y trabajo. Irene Konterllnik y Claudia Jacinto. (Compiladoras) Editorial Losada y UNICEFF. Buenos Aires. 1997

xii Op. Cit.

xiii Op. Cit.

xiv Omar Darío Cardona. "La necesidad de pensar de manera holística los conceptos de Vulnerabilidad y Riesgo. Una crítica y una revisión necesaria para la gestión. Centro de Estudios sobre Desastre y Riesgo (CEDERI). Universidad Andina. Bogotá, 2001

xv Op. Cit. 